\title{
Modelagem 3D e geovisualização aplicada a desastres naturais. Uma proposta de laboratório de ensino e pesquisa para monitoramento e previsão de escorregamentos
}

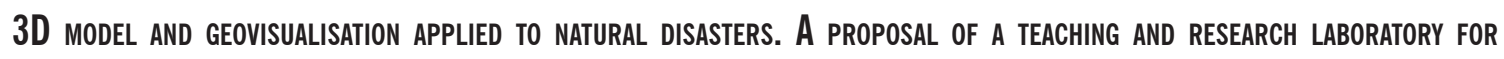
MONITORING AND PREDICTION OF LANDSLIDES

Silvio Jorge Coelho Simões ${ }^{1}$, Márcio Roberto M. de Andrade², Tatiana Sussel Gonçalves Mendes ${ }^{1}$, Rodolfo Moreda Mendes², Luciene Gomes ${ }^{3}$, Cassiano ANTONIO BoRTOLOZO²

\author{
1 Departamento de Engenharia Ambiental, Instituto de Clência e Tecnologia, Universidade Estadual Paulista - UNESP, São José dos Campos/SP, Brasil \\ 2 Centro Nacional de Monitoramento e Alertas de Desastres Naturais - Cemaden Área de Geodinâmica, São José dos Campos, Brasil \\ 3 School of GeOgraphy, UniverSity of LeEDS, LeEds, Reino Unido

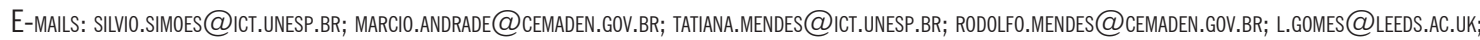 \\ CASSIANOAB@GMAIL.COM
}

Abstract: Landslides have a high degree of uncertainty, requiring new methods for their analysis, monitoring and forecasting. In Brazil, Cemaden is responsible for actions related to natural disasters. Recently, it started, with partner institutions, a project sponsored by FINEP in order to monitor ten landslideprone areas located in different regions of the country. This paper presents the proposal of REDEGEO to implement a modeling and geovisualization laboratory to study landslide processes in urbanized areas. The laboratory consists of three parts: (A) Field surveys to obtain high resolution images from unmanned aerial vehicles, and utilizing geophysics methods (Resistivity and Ground Penetrating Radar - GPR) to obtain internal geometry of outcrops; (B) 3D modeling using the software Geovisionary ${ }^{\circledR}$, which allows the analysis of image and geophysical datasets in different formats, considering their volumetric properties; $(\mathrm{C})$ Geovisualization and Virtual Reality (VR), allowing images obtained in the field to be observed from a human-machine interface, so that researchers can have full immersion in the selected areas. The creation of a laboratory related to natural disasters, including geovisualization and VR capabilities, stimulates the active participation of research teams and creates mechanisms for participation by technology developers, managers, civil defense agents and even the population living in risk-prone areas.
Manuscrito:

Recebido: 13/12/2018

Corrigido: 19/02/2019

Aceito: 04/07/2019

Citação: Simões, S. J.C., Andrade, M. R. M., Mendes, T. S. G., Mendes, R. M., Gomes, L., Bortolozo, C. A. 2019. Modelagem 3D e geovisualização aplicada a desastres naturais. Uma proposta de laboratório de ensino e pesquisa para monitoramento e previsão de escorregamentos. Terræ Didatica, 15, 1-9, e19024. doi: 10.20396/td.v15i0.8654053

Palavras-chave:Landslides, unmanned aerial vehicles, Geophysics, 3D Modeling, virtual reality.

\section{Introdução}

Tradicionalmente, a análise de movimentos de massa em encostas tem utilizado técnicas convencionais de mapeamento, instrumentação e modelagem 2D. Os mapeamentos ditos de "detalhes" envolviam a representação na escala de metros e, muitas vezes, não são suficientes para representar, de forma detalhada, uma determinada superfície (afloramento) em toda a sua heterogeneidade. Além disto, a melhoria dos recursos computacionais para a representação de superfícies do terreno com recursos 3D exige estudos rigorosos da conformação do terreno.

Estas duas últimas décadas viram surgir um significativo crescimento de novos métodos imageadores (de altíssima resolução). Este conjunto de procedimentos se situam no contexto das geotecnologias e, ainda que já viessem sendo utilizadas há determinado tempo, eram aplicadas de forma isolada, sem considerar o enorme potencial integrador destas ferramentas que se complementam na análise geométrica dos afloramentos. O apare- 
cimento de diferentes programas computacionais - com elevados recursos na leitura de dados e para a análise 3D - tem sido o instrumento que tem tornado possível a integração de diferentes tecnologias relacionadas à geometria interna (como o uso de GPR - Ground Penetrating Radar e resistivímetro) e geometria externa (como dados LiDAR - Light Detection And Ranging e de VANT - Veículos Aéreos Não-Tripulados) (Carnet, Massonnet \& King, 1996; Liu, 2008).

No campo das geociências tem surgido uma nova área capaz de integrar todas estas ferramentas designadas de "Geociências Virtuais" que buscam modelar afloramentos para produzir os Modelos Virtuais de Afloramento (MVA) a partir de técnicas de elevada acurácia como LiDAR e Hiperespectral (Buckley, Howell, Enge \& Kurz, 2008; Kurz, Buckley \& Howell, 2013; Souza, 2013) e posteriormente analisadas em software de visualização tridimensional (3D) os quais foram desenvolvidos especificamente para a área de geociências. Inicialmente com aplicações na indústria de óleo e gás (Enge, Buckley, Rotevatn \& Howell, 2007) para produção de MVA de depósitos sedimentares clásticos com potencial de exploração de óleo, atualmente o seu uso tem se estendido para diferentes áreas das geociências como geomorfologia fluvial (Scaioni, Longoni, Melillo \& Papini, 2014) e também para questões envolvendo mudanças globais como variação no nível de geleiras (Nuth, Girod, Kohler, Bahr \& Karlsen, 2016; Weidmann, Jouvet \& Fuck, 2016).

A aplicação das Geociências Virtuais também vem sendo feita na área de desastres naturais como no monitoramento de vulcões (Lev, Hamilton, Scheidt \& Rumpf, 2016) e dos movimentos de massa (Jaboyedoff, Oppikofer \& Abellán, 2012). No caso dos movimentos de massa, a aplicação de tecnologias imageadoras para obtenção de nuvem de pontos permite modelar, com elevada precisão fotogramétrica, possíveis mudanças da morfologia em regiões suscetíveis a escorregamento como aqueles monitorados nos Alpes (Esposito et al 2016; Tesa, Pesci, Ninfo \& Galgaro, 2016). As imagens obtidas a partir dos MVA permitem não apenas sua visualização $2,5 \mathrm{D}$ a partir do monitor, mas possibilitam uma interatividade imersiva entre homem e máquina a partir do se designa genericamente de Realidade Virtual (RV). O termo 2,5 D é oriundo da Computação Gráfica significando duas dimensões e meia sendo aplicado para descrever aqueles fenômenos que aparentam ser tridimensionais mas por alguma razão não o são completamente (por exemplo, não permitem um movimento completamente 3D). Por outro lado RV, conceitualmente, pode ser considerado como um sistema computacional usado para criar um ambiente artificial no qual o usuário tem a impressão de estar dentro deste ambiente, mas com a capacidade de navegar no mesmo interagindo com os objetos de forma intuitiva e natural (Cardoso, Kirner, Lamounier \& Keiner, 2007).

O conceito de RV - integrado a análise geoespacial e ao ambiente SIG (Sistemas de Informação Geográfica) - tem tido um grande avanço em diversos países fazendo com que surjam, grupos que buscam combinar as ferramentas da análise geoespacial com as vantagens de se usar os princípios da geovisualização em ambiente 3D tanto para fins de pesquisa quanto educacional (Faust 1995; Huang, Jiang \& Li, 2010; Feng, 2013). Entretanto, no Brasil, a integração SIG-RV é ainda bastante incipiente com poucos grupos trabalhando nesta interface. No país, o uso de RV tem sido mais direcionado para as áreas de jogos, educação, medicina e engenharia (Cardoso et al, 2007), sem que a noção de geografia e de espaço seja incluída nestas aplicações. Algumas exceções podem ser destacadas como a experiência da Vale, que busca integrar SIG e RV no âmbito das geociências particularmente na área da mineração (Calazans \& Castigliane, 2013) e o trabalho desenvolvido pelo VizLab da Unisinos produzindo afloramento análogo e aplicando para diferentes finalidades ligadas às geociências (Silva, Veronez, Wohnrath, Souza \& Inocêncio, 2014; Silveira, Tognoli, Veronez \& Souza, 2016). Além disto, pode-se mencionar o trabalho de Silva et al (2014) para o imageamento digital e modelagem virtual de rochas carbonáticas voltadas para a indústria de óleo e gás.

Portanto, os registros no país na área de geociências para a interação entre técnicas de campo de alta resolução e modelagem 3D/RV têm sido basicamente nas áreas de mineração e óleo/gás. No caso da área de desastres não tem sido encontrado exemplos de aplicações. Neste contexto, este trabalho busca apresentar uma proposta para a implantação de um laboratório que integre os conceitos de modelos virtuais de afloramento, SIG, modelagem $3 \mathrm{D}$ e realidade virtual e que poderá ter aplicações não apenas em desastres naturais, mas em diferentes áreas da geotecnia. Trata-se de uma proposta inovadora em termos de buscar ir além dos laboratórios tradicionais de geotecnia que fazem a interação entre ensaios de laboratório e modelagem bidi- 
mensional (2D), buscando utilizar os princípios da modelagem 3D e geovisualização para melhorar a representação de situações geotécnicas observadas em campo e os procedimentos da análise de riscos.

A implantação deste laboratório é parte integrante da Rede de Monitoramento Geotécnico (REDEGEO) aprovado pela FINEP e coordenado pelo Centro Nacional de Monitoramento e Alertas de Desastres Naturais (Cemaden) e com a participação do ICT/UNESP, UFPe e UFBa. A parceria Cemaden-UNESP visa, portanto, criar uma concepção nova de laboratório na área de desastres naturais integrando instrumentação de campo, modelagem e geovisualização.

\section{Escopo do projeto e área de estudo}

Cemaden é uma instituição voltada ao desenvolvimento de pesquisas aplicadas em ações preventivas de proteção e defesa civil. Em especial, o Cemaden opera uma ampla rede de sensores tais como pluviômetros, fluviômetros e radares meteorológicos que, auxiliados por modelos de previsão atmosférica, são voltados para emissão de alertas antecipados de inundações e movimentos de massa visando a atender comunidades vulneráveis em áreas de risco geohidrológicos.

O projeto REDEGEO (Andrade \& Mendes, 2016) é uma iniciativa da estratégia nacional de monitoramento em tempo real de fatores ambientais desencadeadores de desastres naturais do Cemaden. O projeto tem como objetivo geral estabelecer uma rede observacional geotécnica que, a médio e longo prazo, possibilite aumentar a precisão dos alertas de movimento de massa emitidos pelo Cemaden e melhor qualificar as ações das Defesas Civis.

Os principais objetivos da REDEGEO seriam: a) instalação e manutenção de uma rede observacional de parâmetros ambientais, neste caso sensores de pluviosi- dade e umidade dos solos acoplados numa plataforma de coleta de dados única (PCDsGEO); b) investigação geológico-geotécnica dos sítios visando à aquisição de dados relacionados aos fatores deflagradores dos deslizamentos e estimativa inicial dos mecanismos dos processos; c) modelagem preditiva com a aplicação de modelos matemáticos de base física e aprimoramento do conhecimento da geodinâmica dos processos; d) ampliação e a difusão das informações através da integração dos modelos de forma imersiva em ambiente 3D.

As áreas pilotos estão localizadas em diferentes domínios geológicos nas regiões sul, sudeste e nordeste, onde o histórico de eventos e desastres de movimentos de massa é significativo. A localização dos dez municípios prioritários selecionados para este projeto é mostrada na Figura 1.

O projeto busca, entre outros objetivos, estudar o tipo de mecanismo de ruptura dos movimentos de massa, os modelos físicos de estabilidade de encostas e estudos de suscetibilidade de terrenos visando determinar os limiares de chuvas e as influências antrópicas nestes processos. Também se pretende elaborar material de apoio à disseminação das informações sobre gerenciamento de risco de deslizamento de encostas.

\section{A proposta do laboratório}

Para atingir aos objetivos do Projeto REDEGEO, seria necessário um vasto conjunto de

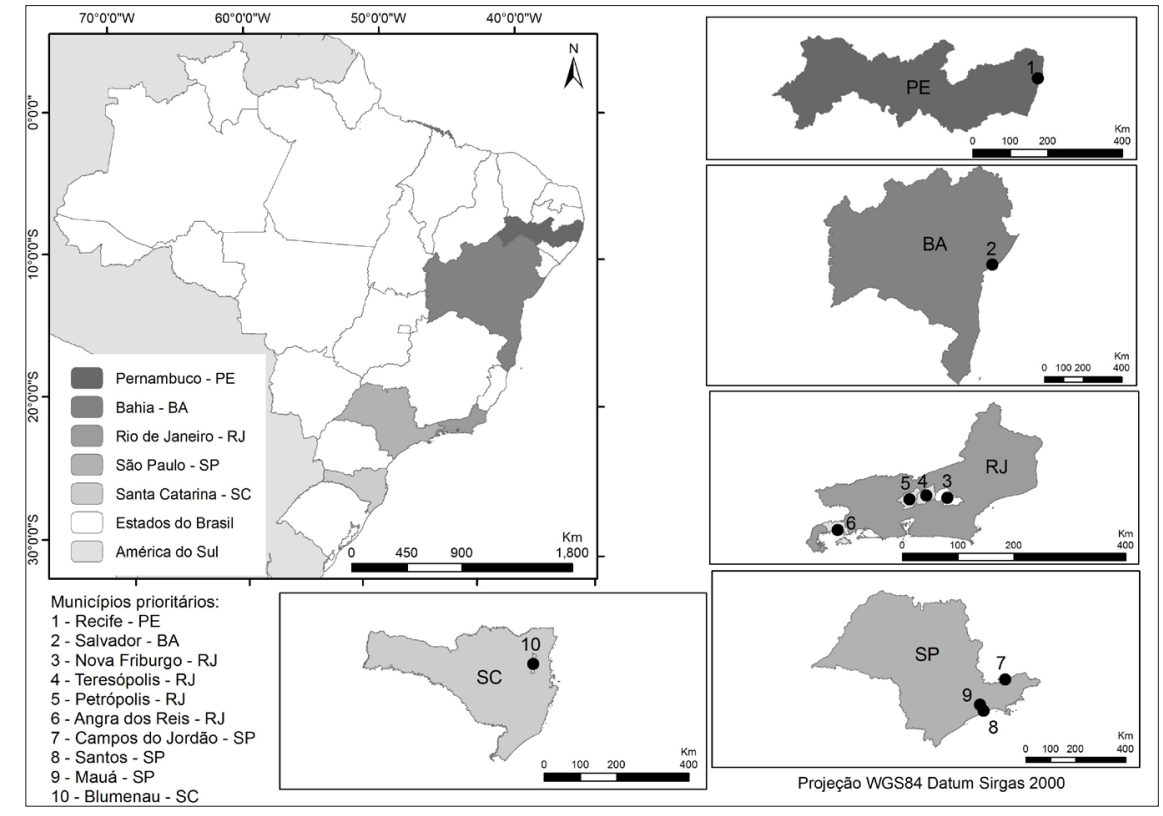

Figura 1. Localização das áreas prioritárias a serem estudadas 
metodologias que incluem instrumentação de campo, de laboratório e de modelagem. Além disto percebeu-se que a utilização da geovisualização associada a métodos que possibilitassem a imersão total dos usuários no ambiente poderia melhorar, significativamente, a análise das áreas submetidas a riscos de desastres. O uso da geovisualização imersiva também poderia ajudar a criar um mecanismo de integração com agentes públicos ligados às áreas de defesa civil, permitindo a visualização, em laboratório, de diferentes situações de riscos normalmente situadas em áreas com elevado nível de antropização.

Desta forma, o laboratório, desenvolvido em uma parceria Cemaden - ICT/UNESP, constaria de três etapas: a) atividades de campo para obtenção de dados da superfície a partir de imageamento externo (uso de imagens de alta resolução) e análise da geometria interna utilizando métodos geofísicos; b) representação, análise e modelagem dos dados de imagens e geofísicos obtidos; c) representação, com o auxílio de RV, das áreas de risco. A Figura 2 mostra, de maneira esquemática, as etapas a serem realizadas.

\section{Levantamentos de campo}

As áreas selecionadas com potencial a movimentos de massa serão representadas da forma mais rigorosa possível que mostre de forma fidedigna a conformação do terreno em superfície e a geometria das camadas em sub-superfície. Como mencionado anteriormente, dois aspectos são considerados: a) imageamento da superfície terrestre e locação e b) geometria interna.

\section{Imageamento e levantamento da superfície terrestre}

A conformação da superfície será representada utilizando-se imagens de satélite com resolução variando de 5 a $30 \mathrm{~m}$ para mapeamentos em escala regional ou local. As imagens obtidas por VANTs

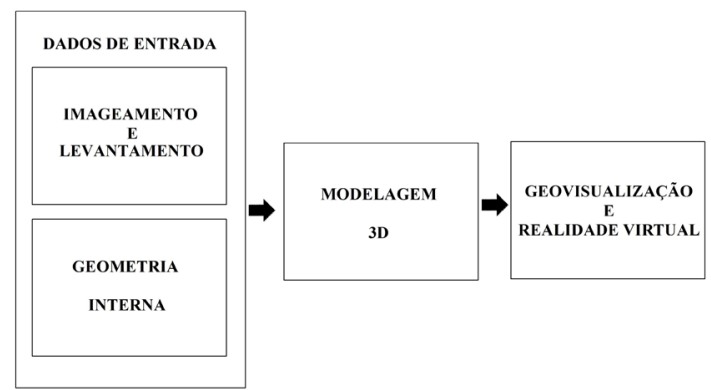

Figura 2. Estrutura do laboratório mostrando as suas três partes constituintes

complementarão as imagens de satélite por sua melhor resolução (em torno de $5 \mathrm{~cm}$ ) além de maior agilidade quando comparado ao uso de aeronaves tradicionais ou da programação do imageamento por satélite. A estação total e o receptor GNSS geodésico com RTK possibilitarão posicionamento, com elevada precisão, dos perfis geofísicos e outras tarefas de campo. A tabela 1 mostra os quatro métodos para o imageamento e levantamento da superfície e, em seguida, se discute cada um deles.

No projeto, as imagens aéreas serão obtidas pela tecnologia VANT, cuja vantagem está na agilidade nas tomadas das imagens e na capacidade de formação estereoscópica. Outra vantagem é em relação ao custo do equipamento, o qual é muito inferior quando se compara com as câmeras fotogramétricas e o sensor LiDAR.

Os VANTs carregam câmeras não métricas e possuem a capacidade de realizar vôos em baixa altitude produzindo imagens de elevada resolução espacial. Para que possa ser gerado o Modelo Digital de Elevação (MDE), o vôo deve ser realizado com sobreposição longitudinal e lateral, da mesma forma como os voos para levantamento aéreo, de modo a gerar pares estereoscópicos. A geração de MDE a partir das imagens baseia-se em algoritmos robustos conhecidos como Structure from Motion Procedures (SfMP) os quais possibilitam o reconhecimento automático de padrões nas imagens e a reconstrução tridimensional do terreno. Nesta etapa de pós-processamento, a aerotriagulação do bloco de

Tabela 1. Métodos de levantamento da superfície utilizados

\begin{tabular}{c|c|c|c}
\hline Método & Resolução & Aplicações & $\begin{array}{c}\text { Custo } \\
\text { relativo }\end{array}$ \\
\hline Imagens de satélite & $30 \mathrm{a} 5 \mathrm{~m}$ & Mapeamento regional \\
\hline $\begin{array}{c}\text { Aerofotos obtidas por } \\
\text { VANTs }\end{array}$ & $\begin{array}{c}\text { até poucos } \\
\text { centímetros }\end{array}$ & $\begin{array}{c}\text { Mapeamento em escala de detalhe } \\
\text { (afloramento) }\end{array}$ & Alto \\
\hline $\begin{array}{c}\text { GNSS Geodésico } \\
\text { (RTK) }\end{array}$ & Milimétrica & $\begin{array}{c}\text { Implantação de rede geodésica de alta precisão } \\
\text { (RTKo }\end{array}$ & Alto \\
\hline
\end{tabular}


imagens é realizada juntamente com calibração da câmera e, em seguida, a nuvem de pontos é gerada baseando-se no conceito de correlação de imagens.

No levantamento por VANT, a utilização de receptor GNSS é imprescindível para o georreferenciamento das imagens. As coordenadas dos pontos de apoio podem ser obtidas com o uso de receptores GNSS por meio do método de posicionamento relativo estático, utilizando uma estação da Rede Brasileira de Monitoramento Contínuo (RBMC) como referência ou por meio da tecnologia RTK (posicionamento cinemático em tempo real). Além disto, o receptor GNSS poderá ser utilizado em levantamentos geofísicos terrestres.

\section{Geometria interna}

Para a análise interna dos afloramentos, foram selecionados dois métodos geofísicos - imageamento geoelétrico e GPR - os quais fornecerão informações complementares dos afloramentos e taludes selecionados por seu histórico de riscos. Estes perfis serão realizados onde preferencialmente existem dados de sondagem a percussão que serão úteis para calibrar e validar os dados obtidos por imageamento geoelétrico e GPR. A tabela 2 compara os dois métodos em termos de resolução, aplicações e custos.

O método da eletrorresistividade busca determinar a distribuição da resistividade elétrica nas estruturas em subsuperfície, tendo como princípio físico a injeção de correntes elétricas (geradas de forma artificial) no solo e a medida das diferenças de potencial geradas por esta injeção. Dessa forma é possível analisar a distribuição das propriedades elétricas da subsuperfície. Dentro do método geoelétrico está a técnica da tomografia elétrica, que é normalmente aplicada em pesquisas que visam a determinar descontinuidades laterais da subsuperfície. Tais descontinuidades são relacionadas a variações na resistividade elétrica dos materiais no subsolo, podendo estar associadas a diferentes materiais geológicos ou variações na composição de uma mesma feição geológica, devido à presença de água, fraturas, composição mineralógica entre outros fatores. O uso de sistemas mais modernos permite a aquisição automática de dados com diferentes arranjos de eletrodos, de forma quase simultânea. Dessa forma é possível obter uma quantidade de dados muito grande em pouco tempo, sendo possível, dessa forma, obter a variação bi e tridimensional da área estudada. Também é possível fazer um acompanhamento da área com a repetição das aquisições ao longo do tempo, gerando assim resultados 4D da resistividade da região.

O GPR é um método ativo, também designado de georadar, que mede a resposta de um alvo em subsuperfície por meio de emissão de ondas eletromagnéticas. Os princípios teóricos e a descrição deste método podem ser encontrados em Gilbert (1999). Ao longo das últimas décadas o GPR vem sendo utilizado nos mais diversos fins em estudos geotécnicos e ambientais. O método se baseia na emissão e reflexão de ondas eletromagnéticas de alta frequência (de $10 \mathrm{MHz}$ até 2,6 GHz), para mapear desde objetos enterrados até estruturas e feições geológicas. O principio físico do método consiste em enviar ondas eletromagnéticas para o subsolo através de uma antena em superfície. Ao encontrar estruturas com diferentes propriedades físicas parte da energia da onda emitida é refletida para a superfície, onde é medida por uma antena receptora. O resultado disso é um radargrama registrado num computador portátil. Os radargramas correspondem a amplitude do sinal refletido em função do tempo de ida e volta do sinal, normalmente apresentados como perfis 2D ou mapas 3D. As frequências das antenas utilizadas impactam na profundidade de investigação, sendo que antenas com frequências mais altas possuem melhor resolução, mas menor profundidade de investigação. O meio geológico estudado também influencia diretamente a profundidade de investigação, com camadas muito condutivas agindo como atenuadoras do sinal e impedindo a propagação das ondas em camadas mais profundas.

O uso da tomografia elétrica para estudos de desastres naturais é muito disseminado no mundo todo, com muitos trabalhos na área de deslizamentos de encostas. No trabalho de Perrone,

Tabela 2. Métodos para representação da geometria interna

\begin{tabular}{l|l|l|c}
\hline \multicolumn{1}{c|}{ Método } & \multicolumn{1}{c|}{ Resolução } & \multicolumn{1}{c}{ Aplicações } & Custo relativo \\
\hline Imageamento Geoelétrico & Métrica & $\begin{array}{l}\text { Imageamento do arcabouço interno do } \\
\text { afloramento }\end{array}$ & Médio \\
\hline GPR & Centimétrica & $\begin{array}{l}\text { Imageamento do arcabouço interno do } \\
\text { afloramento }\end{array}$ \\
\hline
\end{tabular}


Lapenna \& Piscitelli (2014) é feita uma revisão bastante abrangente de trabalhos nesse sentido, mostrando as capacidades do método para tal. O GPR também é muito utilizado em trabalhos de desastres naturais, como exemplo, em Lissak, Maquaire, Malet, Lavigne \& Gomez (2015), onde o GPR foi utilizado para estimar movimentos verticais de escorregamentos rotacionais, combinados com levantamentos geodésicos de alta precisão. O método também foi utilizado no trabalho de Roch, Chwatal \& Brückl (2006) para determinar áreas mais propensas a quedas de blocos de rocha na Áustria. Os métodos eletrorresistivo e GPR também podem e são normalmente utilizados de forma conjunta para um melhor imageamento da subsuperfície estudada. O uso da técnica conjunta de tomografia elétrica e GPR também esta sendo muito utilizada para monitorar o deslocamento de escorregamentos. Trabalhos como os de Sass, Bell \& Glade (2008) e Bichler et al (2004) mostram a grande qualidade dos resultados obtidos com os métodos nesse tipo de situação. Como resultados desses trabalhos são obtidos mapas 2D ou 3D com as interfaces geológicas mais rasas e a distribuição da resistividade elétrica da área.

\section{Modelagem 3D}

Para a produção de modelos em 3D se optou pelo software Geovisionary desenvolvido pela empresa Vitalis em parceria com o British Geological Survey (BGS). O Geovisionary é um conjunto de softwares desenvolvido especificamente para reconhecimento virtual de campo em modelos 3D. O software é capaz de visualizar dados espaciais de alta resolução (provenientes das tecnologias LiDAR, VANT e de dados geofísicos) que contenham feições geomorfológicas de alta precisão em taludes. Desta forma permite, por exemplo, observar mudanças bruscas de declividade as quais podem ser digitalizadas diretamente em um MDE 3D. Por outro lado, normalmente escorregamentos tendem a apresentar heterogeneidade vertical e lateral dos parâmetros físicos presentes no material (rocha e/ ou solo), daí a importância de se utilizar a análise 3D. Em áreas suscetíveis a escorregamentos no Reino Unido, Merrit et al (2013) utilizam o software Geovisionary para integrar dados geofísicos, dados de sensoriamento remoto LiDAR e métodos geotécnicos para identificar áreas de escorregamentos que são recentemente mais ativas. No caso de dados de eletrorresistividade, estes podem ser apresenta- dos de forma volumétrica a partir de dados de alta resolução permitindo identificar o contraste e a variação litológica dos perfis.

\section{Geovisualização e RV}

Como quarta etapa do laboratório pretende-se estabelecer um empreendimento piloto de geovisualização em RV voltado para a área de desastres naturais com dois objetivos fundamentais: explorar o potencial desta tecnologia para a visualização em detalhe das áreas suscetíveis a riscos geológicos a escorregamentos e criar uma interface de comunicação entre pesquisadores, gestores e membros da defesa civil dos municípios. Estão disponíveis no mercado diferentes tipos de soluções em RV, desde equipamentos de alto custo (como cavernas digitais), para o uso limitado de pessoas, até a visualização de custo relativamente menor, a partir de projeções planas que podem ser realizadas em salas especialmente preparadas e que podem receber dezenas de pessoas. Com o advento da Web e da redução de custos dos equipamentos, o uso de RV passou a ter uma grande disseminação e simplificou bastante a interação homem-máquina e as possibilidades de imersão dentro dos ambientes virtuais.

No caso do laboratório que se pretende implantar optou-se por uma solução de visualização de custo relativamente baixo baseado em projeção plana em um espaço que permita integrar o seu uso para pesquisa em desastres naturais assim como criar um canal de conexão com setores da sociedade como as defesas civil municipais recriando, nas áreas suscetíveis a desastres, ambientes próximos do real.

A sala está fundamentada em uma solução integrada desenvolvida pelo BGS em parceria com a empresa Virtalis, que desenvolveram uma solução conjunta integrando o software Geovisionary a um sistema de RV que inclui, entre seus principais componentes, projetor 3D, tela de projeção plana, e óculos/mouse 3D. Soluções semelhantes a esta foi adotada no Brasil pela Vale com aplicações em mineração (Calazans \& Castiglione, 2013). Na área de desastres naturais não se conhece aplicação de RV no país. De fato, o conceito de RV associado à noção de geografia (ou espaço) tem pouco desenvolvimento no Brasil.

De uma maneira geral, RV pode ser entendida como uma forma de "interface homem-computador" que, no caso aqui discutido, envolve o "ambiente geográfico". Segundo Brodlie et al 
(2002) o conceito de RV é distinto das tradicionais "transformações cartográficas" pois a ênfase na RV é no processo de ligação entre a representação (mapa) e o usuário, e não na representação em si como ocorre na cartografia.

A RV não é um processo único, mas pode ser vista como um contínuo baseado em diferentes níveis de interações e graus de imersão. O que se mede é o grau de affordance (termo ainda sem correspondente no português). Affordance mede o quanto um objeto pode ser reconhecido por um indivíduo sem a necessidade de uma prévia explicação (Gibson, 1979). Quanto maior a affordance maior será a identificação do seu uso e tem sido aplicado para diferentes áreas entre elas a interação homem-computador (IHC) e inteligência artificial. A Figura 3 mostra como ocorre a evolução entre homem e máquina e como a representação geográfica em formas $3 \mathrm{D}$ pode interagir em diferentes níveis. Em seu nível mais baixo (nível a) a interação homem-máquina é muito baixa; no nível $b$ existe uma certa affordance espacial do mundo real. Neste nível, a ferramenta on the fly disponível em alguns SIGs, como 3DAnalyst/ArcGIS, IDRISI, são exemplos deste nível de interação. No nível $c$ a interação homem-máquina aumenta e começa a ocorrer um forte sentido de imersão. A acurácia dos MDE e os recursos 3D dos softwares permitem a representação de paisagens detalhadas onde a sensação de imersão aumenta. No nível mais avançado (nível $d$ ) o usuário é total e fisicamente imerso no ambiente e responde como se operasse no mundo real dentro de um ambiente virtual muito sofisticado.

Portanto trabalhar com a relação Geografia e RV se fazem com diferentes níveis de imersão na relação homem-máquina. No caso do laboratório que se pretende implantar, o nível de imersão é total no sentido de se obter completa imersão no ambiente analisado.

A interação entre Geografia e RV em um nível em que se alcance a imersão total (nível d, Figura 3) é relativamente pouco desenvolvida no Brasil. Normalmente as comunidades ou grupos de pesquisa que trabalham com RV desconhecem os fundamentos de cartografia e os princípios de análise geoespacial e, por outro lado, os grupos que trabalham com análise geoespacial normalmente vão até o nível c (Figura 3) utilizando modelagem 3D mas sem considerar a criação de ambientes com imersão total (nivel $d$, da Figura 3).

Nos países desenvolvidos, a relação SIG-RV tem crescido muito nas últimas duas décadas par-

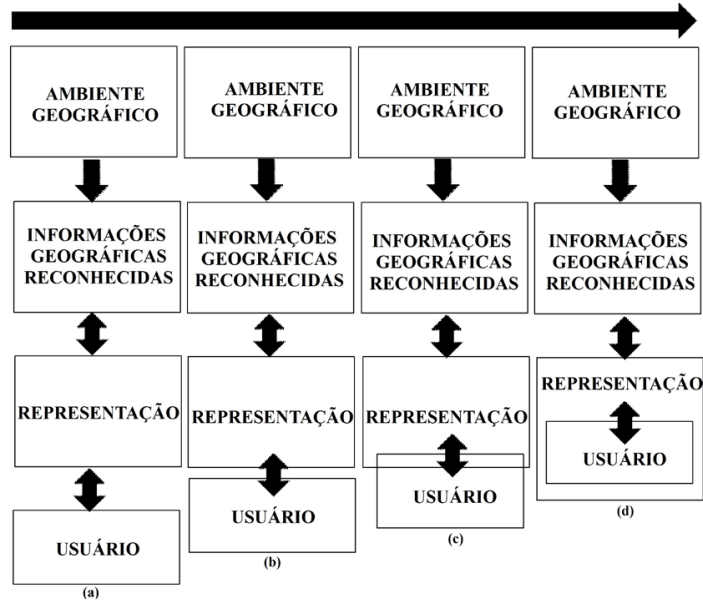

Figura 3. Diferentes níveis de interação homem-máquina

ticularmente nos Estados Unidos, Reino Unido, Holanda, Alemanha, Japão e, mais recentemente, a China conforme se pode observar em duas publicações de divulgação sobre o tema (Fisher \& Unwin, 2002; Lin \& Batty, 2012).

O crescimento da interação entre Geografia e RV está criando uma nova área designada de Virtual Geographic Environments - VGE (Ambientes Geográficos Virtuais) que leva em conta os fundamentos dos SIG e da ciência da informação geográfica considerando, especialmente, a maneira como os usuários e softwares interagem. Os fundamentos e aplicações da VGE estão bem descritos por Lin \& Batty (2012). Outro conceito muito próximo em termos de significado corresponde a geovisualização que, segundo Cai (2012), trata dos desafios na interação SIG-homem e em aspectos relacionados à cognição humana. Ainda segundo este autor, a geovisualização começa a ganhar status de uma nova ciência integrados a uma área maior designada de pesquisa visual analítica, mas com uma abordagem restrita aos dados geoespacias.

Dentro de uma conotação mais geológica, recentemente, tem sido cunhado o termo Geociências Virtuais (Buckley, Naumann \& Eide, 2016) que busca integrar levantamentos detalhados de campo (por exemplo, dados do sistema LiDAR, de VANT e GPR) com a melhoria dos processos de modelagem 3D e geovisualização. Inicialmente, associado a exploração dos recursos naturais - como gás/petróleo e mineração - e atualmente envolvendo diferentes áreas, com destaque para a área de desastres naturais.

Portanto, a fundamentação teórica da proposta do laboratório se insere nos princípios destas três novas áreas de conhecimento (Ambientes Geográ-

\begin{tabular}{c|c|c|c|c|c}
\hline (C) Terrae Didat. & Campinas, SP & v.15 & $1-9$ & e019024 & 2019 \\
\hline
\end{tabular}


ficos Virtuais, Geovisualização e Geociências Virtuais) no sentido de que a compreensão e a nossa capacidade de prever desastres naturais (como os escorregamentos) depende, por um lado, de se utilizar métodos de campo com elevada acurácia que permita detectar heterogeneidades geológicas e geomorfológicas e, por outro, melhorar os sistemas de visualização, que permitam compreender a complexidade dos terrenos em escala de afloramento.

\section{Conclusões}

Os processos responsáveis pelos movimentos de massa são muito complexos e envolvem elevado grau de incerteza. Desta maneira a integração de métodos e técnicas que permitam o detalhamento da superfície topográfica (como os levantamentos por VANT e/ou LiDAR) juntamente com o melhor conhecimento da diversidade litológica e de micro-fraturas (como eletro-resistividade e GPR) são procedimentos que podem melhorar a compreensão dos processos responsáveis pelos escorregamentos. Os softwares de modelagem 3D tiveram grande avanço e permitem a análise volumétrica com muitos recursos associados. A geovisualização, associada à RV com imersão total, teve uma grande redução de custos nos últimos anos e permite que situações observadas no campo possam ser transpostas para o laboratório, tornando possível que pesquisadores de diferentes formações possam avaliar um determinado problema em condições muito próximas das reais.

A ideia de um laboratório que inclua geovisualização com RV em um ambiente de tela plana estimula a participação da equipe e cria mecanismos para a participação da sociedade. Portanto, a utilização da RV realidade virtual com imersão em 3D permite a difusão do conhecimento entre pesquisadores, desenvolvedores de tecnologias, gestores, agentes de defesa civil e população das áreas de risco. Desta forma, possibilita uma significativa contribuição para o gerenciamento do risco de deslizamentos de encostas/taludes, considerando tanto uma melhor compreensão das causas dos movimentos de massa, quanto ao estabelecimento de planos de contingência.

\section{Referências}

Andrade, M. R. M., \& Mendes, R. M. (Coords.). (2016). Subprojeto REDEGEO - Rede de Monitoramento Geotécnico. In: Angelis, C. F. (Co- ord.). 2016. Projeto REMADEN - Rede Nacional de Desastres Naturais. Cemaden: Centro Nacional de Monitoramento e Alertas de Desastres Naturais/ Ministério de Ciência, Tecnologia, Inovações e Comunicações, FINEP-Financiadora de Estudos de Projetos, FNDCT-Fundo Nacional de Desenvolvimento Científico e Tecnológico. Carta Convite MCTI/FINEP/FNDCT 01/2016.

Bichler, A., Bobrowsky, P., Best, M., Douma, M., Hunter, J., Calvert, T., \& Burns, R. (2004). Threedimensional mapping of a landslide using a multigeophysical approach: the Quesnel Forks landslide. Landslides, 1(1), 29-40.

Brodlie, K., Dykes, J., Gillings, M., Haklay, M. E., Kitchin, R., \& Kraak, M-J. (2002). Geography in VR: context. In: P. Fischer, D. Unwin (Ed.). Virtual Reality in Geography. New York, NY: Taylor \& Francis. p. 7-16.

Buckley, S. J., Naumann, N. K., \& Eide, C. H. (Eds.). (2016). $2^{\text {nd }}$ Virtual Geoscience Conference, Proceedings. Bergen, Norway: Uni Research CIPR.

Buckley, S. J., Howell J. A., Enge H. D., \& Kurz T. H. (2008). Terrestrial laser scanning in geology: data acquisition, processing and accuracy considerations. Journal of the Geological Society, 165(3), 625638.

Cai, G. (2012). Modeling contextual knowledge for adaptive geographic visualization. In: H. Lin \& M. Batty (Ed.). Virtual Geographic Environments (pp. 151-163). Redlands, ESRI Press.

Calazans, P. P., \& Castiglione, L. H. G. (2013). Produção geoinformacional e geovisualização para apoio a planejamento e projeto: um empreendimento piloto em realidade virtual. Anais XVI Simpósio Brasileiro de Sensoriamento Remoto - SBSR. Foz do Iguaçu, Brasil: INPE.

Cardoso, A., Kirner, C., Lamounier, E. \& Keiner, J. (2007). Tecnologias para o desenvolvimento de sistemas de realidade virtual e aumentada. Recife: Editora da UFPE.

Carnet, C., Massonnet, D., \& King, C. (1996). Two examples of the use of SAR interferometry on displacement fields of small spatial extent. Geophysics Research Letters, 23 (24), 3579-3582.

Enge, H. D., Buckley, S. J., Rotevatn, A., \& Howell, J. A. (2007). From outcrop to reservoir simulation model: workflow and procedures, Geosphere, 3 (6), 469-490.

Esposito, G., Salvini, R., Danzi, M., Matano, F., Sachi, M., Seddaiu, M., S...., \& Natale, G. (2016). 3D change detection analysis of a coastal landslide performed by multi-temporal point cloud comparison. Proceeding of II Virtual Geoscience Conference. Bergen, Norway.

Faust, N. L. (1995). The virtual reality of GIS. Environment and Planning. Planning and Design, 22, 257-268. 
Feng, J. (2013). Virtual reality: an efficient way in GIS class teaching. International Journal of Computer Science, 10 (1), 363-367.

Fisher, P. \& Unwin, D. (Eds). (2002). Virtual reality in Geography. London, England: Taylor \& Francis.

Gibson, J. J. (1979). The ecological approach to visual perception. Nova York, NY: Lawrence Erlbaum Association.

Gilbert, R. (1999). A handbook of geophysical techniques for geomorphic and environmental research. Ottawa: Geological Survey of Canada.

Huang B., Jiang, B., \& Li, H. (2010). An integration of GIS, virtual reality, and the Internet for vi,sualization, analysis and exploration of spatial data. International Journal of Geographical Information Science 15, 439-456.

Jaboyedoff, M., Oppikofer, T., \& Abellán, A. (2012). Use of LIDAR in landslide investigation: A review. Natural Hazards, 61 (1), 5-28.

Kurz, T. H., Buckley, S. J., \& Howell, J. A. (2013). Close-range hyperspectral imaging for geological field studies: workflow and methods. International Journal of Remote Sensing, 34 (5), 1798-1822.

Lev, E., Hamilton, C. W., Scheidt, S. P., Rumpf, M. E. (2016). Mapping lava flow morphology and structure with unmanned aerial vehicles. Proceeding of II Virtual Geoscience Conference. Bergen, Norway.

Lin, H. \& Batty, M. (Eds) (2012). Virtual Geographic Environments. Redlands: ESRI Press.

Lissak, C., Maquaire, O., Malet, J. P., Lavigne, F., \& Gomez, D. R. 2015. Ground-penetrating radar observations for estimating the vertical displacement of rotational landslides. Natural Hazards and Earth Systems Sciences, 15, 1399-1406.

Liu, X. (2008). Airborne LiDAR for DEM generation: some critical issues. Progress Physical Geography, 32, 31-49.

Merrit, A. J., Chambers, J. E., Murphy, W., Wilkinson, P. B., West, L. J., Gunn, D. A., Meldrun, P. I., Kirkham, M., \& Dixon, N. (2014). 3D Ground model development for an active landslide in Lias mudrocks using geophysical, remote sensing and geotechnical methods. Landslides, 11, 537-550.
Nuth, C., Girod, L., Kohler, J., Bahr, K., \& Karlsen, T. I. (2016). Detailed glacier crevasse morphology mapped by helicopter. Proceeding of II Virtual Geoscience Conference. Bergen, Norway.

Perrone, A., Lapenna, V., \& Piscitelli, S. (2014). Electrical resistivity tomography technique for landslide investigation: A review. Earth-Science Reviews, 135, 65-82.

Roch, K. H., Chwatal, W., \& Brückl, E. (2006). Potentials of monitoring rock fall hazards by GPR: Considering as example the results of Salzburg. Landslides, 3 (2), 87-94.

Sass, O., Bell, R., \& Glade, T. (2008). Comparison of GPR, 2D-resistivity and traditional techniques for the subsurface exploration of the Öschingen landslide, Swabian Alb (Germany). Geomorphology, 93 (1), 89-103.

Scaioni, M., Longoni, L., Melillo, V., \& Papini, M. (2014). Remote sensing for landslide investigations: An overview of recent achievements and perspectives. Remote Sensing, 6, 9600-9652.

Silva, R. M., Veronez, M. R., Wohnrath, F. M., Souza, M. K., \& Inocêncio, L. C. (2014). Accuracy Analysis of Digital Outcrop Models Obtained from Terrestrial Laser Scanner (TLS) International Journal of Advanced Remote Sensing and GIS, 3(1), 506-515.

Silveira, L. G., Tognoli, F. M. W., Veronez, M. R., \& Souza, M. K. (2016). An algorithm for automatic detection and orientation estimation of planar structures in LiDAR-scanned outcrops. Computers E Geosciences, 1, 1.

Souza, A. M. (2013). Proposta metodológica para o imageamento, caracterização, parametrização e geração de modelos virtuais de afloramentos. Tese de Doutorado. Rio Grande do Norte, Brasil: UFRN, Centro de Ciências Exatas e da Terra.

Tesa, G., Pesci, A., Ninfo, A., \& Galgaro, A. (2016). Fast surveying of a sea cliff and a landslide based on structure from motion photogrammetry. Proceedings of II Virtual Geoscience Conference, Bergen, Norway.

Weidmann, Y., Jouvet, G., \& Fuck, M. (2016). Multi-temporal UAV-survey of a calving glacier in Northwest Greenland. Proceeding of II Virtual Geoscience Conference, Bergen, Norway. 